\title{
H-16. Continuous Observation of Cerebrospinal Fluid Pressure by Auto-recording System
}

Hiroshi Sugrura, Toru Yoshida

Susumu Kato and Kentaro Mimatsu

Orthopedic Division, Kariya-Toyota Hospital 